\title{
Influence of Salmonella enterica serovar Enteritidis infection on the composition of chicken cecal microbiota
}

\author{
Petra Videnska, Frantisek Sisak, Hana Havlickova, Marcela Faldynova and Ivan Rychlik
}

\begin{abstract}
Background: Infection of newly hatched chicks with Salmonella enterica serovar Enteritidis (S. Enteritidis) results in an inflammatory response in the intestinal tract which may influence the composition of gut microbiota. In this study we were therefore interested whether $S$. Enteritidis induced inflammation results in changes in the cecal microbiota. To reach this aim, we compared the cecal microbiota of non-infected chickens and those infected by $\mathrm{S}$. Enteritidis by pyrosequencing the V3N4 variable regions of genes coding for $16 \mathrm{~S}$ rRNA.

Results: Cecal microbiota of chickens up to 19 days of life was dominated by representatives of Enterobacteriaceae, Lachnospiraceae and Ruminococcaceae, followed by Lactobacillaceae. The presence of Lachnospiraceae did not change after $S$. Enteritidis infection. Enterobacteriaceae increased and Ruminococcaceae decreased after $S$. Enteritidis infection in two independent experiments although these results were not significant. A significant increase in both experiments was observed only for the representatives of Lactobacillaceae which may correlate with their microaerophilic growth characteristic compared to the obligate anaerobes from the families Lachnospiraceae and Ruminococcaceae.
\end{abstract}

Conclusions: We conclude that $S$. Enteritidis infection influences the composition of the cecal microbiota in chickens but these changes are minor in nature and should be understood more as an indirect consequence of infection and inflammation rather than a positively selected evolutionary trait.

Keywords: Chicken, Microbiome, Intestinal tract, Pyrosequencing, Salmonella

\section{Background}

Gut microbiota plays an important role in shaping the host's immune response, nutrient uptake and production of metabolites essential for the host [1-4]. The composition of gut microbiota is not constant and develops over time. In warm blooded vertebrates, the initial gut colonizers are those belonging to the phylum Proteobacteria, particularly the family Enterobacteriaceae [5,6]. Later in life, representatives from the phylum Firmicutes consisting of the families Lachnospiraceae, Ruminococcaceae, Clostridiaceae or Lactobacillaceae dominate and in older animals, the digestive tract becomes populated with representatives from the phylum Bacteroidetes [5-7]. Besides the above mentioned life stage factor, the composition of gut microbiota is shaped by food or feed composition and, of course, is

\footnotetext{
* Correspondence: rychlik@vri.cz

Veterinary Research Institute, Brno, Czech Republic
}

highly responsive to extreme interventions like antibiotic therapy [8-11].

The composition of gut microbiota may also change during various diseases which are associated with inflammation and an influx of phagocytes and lymphocytes from the circulation [12-14]. Changes in the composition of gut microbiota caused by inflammation are observed also in animals or humans infected with non-typhoid serovars of Salmonella enterica [15-18]. An influx of phagocytes into the inflamed intestinal tract following S. enterica infection results in the production of antimicrobial metabolites such as proteases, reactive oxygen species, nitric oxide radicals and chelators of bacterial siderophores. Some of these antimicrobial products result not only in pathogen inactivation but also in damage to the host's own tissue, particularly damage to the integrity of the intestinal epithelium and efflux of electrolytes clinically manifesting as diarrhea. It

\section{Biomed Central}


is therefore legitimate to hypothesize that the pathogen inducing the inflammation should be adapted to life in such an environment and the ability to survive in inflamed tissue is considered as an evolutionary adaptation also in Salmonella [19]. Lipocalin 2 produced by murine neutrophils in the intestine of streptomycin-treated mice in response to $S$. enterica infection binds to enterochelin, a type of bacterial siderophore. However, S. enterica produces glycosylated forms of enterochelin and this type of siderophore is not captured by lipocalin 2 . In this way, S. enterica gets a growth advantage in the inflamed intestine over the microbiota members expressing the non-glycosylated forms of enterochelins [20]. An additional advantage $S$. enterica has over the other microbiota members is its ability to respire tetrathionate. Although this electron acceptor is absent from a normal, healthy intestinal tract, it is produced in the inflamed intestine from common reduced sulfur compounds by oxidative species produced by infiltrating neutrophils [21]. However, it is unknown to what extent these S. enterica adaptations will result in changes to the composition of gut microbiota.

Infection of newly hatched chicks with Salmonella enterica serovars Enteritidis or Typhimurium ( $S$. Enteritidis or $S$. Typhimurium) results in an inflammatory response in the intestinal tract [22-24]. We were therefore interested whether the inflammation induced by Salmonella infection will or will not affect the development and composition of gut microbiota in chickens. Our previous results using T-RFLP and quantitative real time PCR indicated that the changes in gut microbiota in chickens are not as dramatic as one would expect from data in other models of host - pathogen interactions [12-14,16]. In this study we have therefore used pyrosequencing of V3 and V4 variable regions of $16 \mathrm{~S}$ rRNA genes to characterize the consequences of $S$. Enteritidis infection on the composition of chicken cecal microbiota in detail. Although we identified species which decreased or increased after the infection, overall we did not detect any large scale changes indicating that modification and overgrowth of the cecal microbiota is not a major driving force in the evolution of Salmonella - chicken interactions.

\section{Results}

Altogether, 362609 reads were analyzed in this study following pyrosequencing the amplification products of the V3/V4 hypervariable $16 \mathrm{~S}$ rRNA genes. These reads were distributed among 2079 different OTUs (Operational Taxonomic Unit) identified in 30 samples from the noninfected chickens and 20 samples from $S$. Enteritidis infected chickens (we failed with pyrosequencing of one sample originating from a 19-day-old chicken infected with $S$. Enteritidis on day 16). Number of reads per sample ranged from 1,141 to 21,109 (see Table 1 and Additional file 1). The composition of cecal microbiota was similar to that reported previously for young chickens [5,7,16,25]. The cecal microbiota in young chickens was dominated by representatives of the family Lachnospiraceae (phylum Firmicutes) followed by Ruminococcaceae (Firmicutes) and Enterobacteriaceae (Proteobacteria). The presence of the predominant family Lachnospiraceae was unaffected by $S$. Enteritidis infection. Infection of chickens with $S$. Enteritidis (see Table 2 for $S$. Enteritidis counts) caused a minor numerical increase in Enterobacteriaceae in the microbiota of infected chickens at the expense of Ruminococcaceae in both experiments. In addition, although forming a minority population in the cecum, representatives of Lactobacillaceae repeatedly increased after the infection with $S$. Enteritidis (Figure 1).

In the next analysis we compared whether $S$. Enteritidis infection affected the total complexity of chicken cecal microbiota characterized by the number of different OTUs predicted as Chaol estimates. Rather unexpectedly, infection of 4, 7, 10, 13 and 16-day-old chickens with $S$. Enteritidis did not result in reproducible changes in the number of OTUs in the cecum. This was confirmed also in the repeated experiment in which luminal and mucus-associated microbiota were characterized separately (Figure 2).

Since the total number of OTUs may not properly characterize the microbial populations in the cecum of $S$. Enteritidis infected or non-infected chickens, the microbiota of individual chickens was compared further by UniFrac analysis followed by PCoA. PCoA clustered chicken microbiota from the two experiments into separate clusters. However, no obvious clustering of the infected and non-infected chickens was recorded in either of the experiments (Figure 3).

The contradictory results presented in Figures 1, 2 and 3 , could be influenced by the fact that representatives of the predominant family Lachnospiraceae were not affected by $S$. Enteritidis thus masking the effect of $S$. Enteritidis infection on the minority populations. Finally we therefore analyzed individually all 2079 different OTUs for significant changes in response to $S$. Enteritidis infection by t-test considering the comparisons with $\mathrm{p}<0.05$ as significant. In experiment 1 , none of the OTUs significantly decreased after $S$. Enteritidis infection. On the other hand, six OTUs significantly increased after $S$. Enteritidis infection. Three of them belonged to the family Enterobacteriaceae (one of them being Salmonella), and the remaining ones belonged to the families Lachnospiraceae, Lactobacillaceae and Bifidobacteriaceae. Five of these OTUs formed less than $0.02 \%$ of the total microbiota in the non-infected chickens and still less than $0.1 \%$ after infection. The only OTU which significantly increased after the infection forming around $1 \%$ of total microbiota was the 
Table 1 Basic characteristics of chicken cecal microbiota samples for individual time points shown as average \pm SD out of 3 independent chickens

\begin{tabular}{|c|c|c|c|c|c|c|}
\hline Experiment 1 & $\mathrm{NI} 4^{*}$ & NI7 & NI10 & $\mathrm{NI13}$ & NI16 & NI19 \\
\hline No. reads & $2409 \pm 265$ & $4198 \pm 1570$ & $3314 \pm 1097$ & $7577 \pm 455$ & $14994 \pm 2354$ & $9586 \pm 2045$ \\
\hline Observed species & $54 \pm 6$ & $107 \pm 18$ & $94 \pm 19$ & $153 \pm 33$ & $307 \pm 42$ & $233 \pm 28$ \\
\hline Chaol estimate & $69 \pm 8$ & $199 \pm 81$ & $146 \pm 40$ & $276 \pm 88$ & $605 \pm 103$ & $418 \pm 79$ \\
\hline Shannon index & $3.15 \pm 0.33$ & $4.33 \pm 0.20$ & $4.54 \pm 0.02$ & $4.78 \pm 0.26$ & $5.39 \pm 0.22$ & $5.42 \pm 0.11$ \\
\hline Simpson index & $0.82 \pm 0.04$ & $0.91 \pm 0.01$ & $0.94 \pm 0.00$ & $0.93 \pm 0.02$ & $0.96 \pm 0.00$ & $0.96 \pm 0.00$ \\
\hline Experiment 1 & & S7 & S10 & S13 & $\mathrm{S} 16$ & S19 \\
\hline No. reads & & $9940 \pm 3942$ & $16514 \pm 2740$ & $12725 \pm 3783$ & $5840 \pm 921$ & $17992 \pm 3235$ \\
\hline Observed species & & $155 \pm 81$ & $187 \pm 8$ & $196 \pm 47$ & $149 \pm 5$ & $479 \pm 11$ \\
\hline Chao1 estimate & & $277 \pm 98$ & $298 \pm 66$ & $289 \pm 76$ & $265 \pm 36$ & $846 \pm 80$ \\
\hline Shannon index & & $4.15 \pm 0.36$ & $4.61 \pm 0.18$ & $4.53 \pm 0.39$ & $5.05 \pm 0.37$ & $5.55 \pm 0.14$ \\
\hline Simpson index & & $0.89 \pm 0.02$ & $0.92 \pm 0.02$ & $0.92 \pm 0.03$ & $0.94 \pm 0.02$ & $0.96 \pm 0.00$ \\
\hline Experiment 2 & Lumen N7 & Lumen N10 & Lumen S10 & Mucus NI7 & Mucus NI10 & Mucus S10 \\
\hline No. reads & $3302 \pm 391$ & $6958 \pm 4142$ & $4107 \pm 3942$ & $1307 \pm 161$ & $4278 \pm 79$ & $1826 \pm 404$ \\
\hline Observed species & $184 \pm 33$ & $221 \pm 24$ & $196 \pm 81$ & $95 \pm 5$ & $132 \pm 15$ & $87 \pm 10$ \\
\hline Chao1 estimate & $283 \pm 76$ & $319 \pm 42$ & $298 \pm 98$ & $150 \pm 21$ & $204 \pm 26$ & $110 \pm 2$ \\
\hline Shannon index & $5.24 \pm 0.31$ & $4.98 \pm 0.43$ & $5.00 \pm 0.36$ & $4.82 \pm 0.07$ & $4.64 \pm 0.08$ & $4.63 \pm 0.26$ \\
\hline Simpson index & $0.95 \pm 0.01$ & $0.93 \pm 0.03$ & $0.93 \pm 0.02$ & $0.93 \pm 0.01$ & $0.93 \pm 0.00$ & $0.92 \pm 0.02$ \\
\hline
\end{tabular}

* $\mathrm{NI}$ - non-infected chicken followed by age of chickens in days; S - Salmonella Enteritidis infected chickens followed by age of chickens in days, Lumen and Mucus - luminal and mucus associated microbiota characterized separately in experiment 2.

representative of Lactobacillaceae. This OTU formed $0.24 \%$ of the total microbiota in the cecum of noninfected chickens and increased to $1.10 \%$ after infection with $S$. Enteritidis (Figure 4). RDP seqmatch analysis showed that the rRNA sequence of this OTU was identical to Lactobacillus ultunensis.

In experiment 2, six OTUs significantly decreased and 2 OTUs significantly increased after $S$. Enteritidis infection. Five of the OTUs that decreased after infection belonged to the family Lachnospiraceae and the remaining one belonged to the family Streptococcaceae. Two OTUs which significantly increased after $S$. Enteritidis infection belonged to families Lactobacillaceae and Ruminococcaceae. As in experiment 1, only the representative of Lactobacillaceae formed more than $1 \%$ of the total microbiota, specifically

Table 2 S. Enteritidis colonization of the caecum of chickens after oral infection

\begin{tabular}{ccc}
\hline & \multicolumn{2}{c}{$\log$ CFU/g of caecum } \\
\cline { 2 - 3 } Age (days)* & Exp1 & Exp2 \\
\hline 7 & $7.96 \pm 0.35$ & $\mathrm{ND}$ \\
10 & $7.96 \pm 0.22$ & $6.73 \pm 0.06$ \\
13 & $8.13 \pm 0.08$ & $\mathrm{ND}$ \\
16 & $7.57 \pm 0.49$ & $\mathrm{ND}$ \\
19 & $7.44 \pm 0.82$ & $\mathrm{ND}$ \\
\hline
\end{tabular}

*age of chickens means age when the chickens were sacrificed, i.e. the chickens were therefore infected 3 days earlier. ND not done.
$1.70 \%$ in the cecal microbiota of the non-infected chickens and $10.44 \%$ after $S$. Enteritidis infection (Figure 4). RDP seqmatch analysis showed that the rRNA sequence of this OTU was identical to Lactobacillus gasseri.

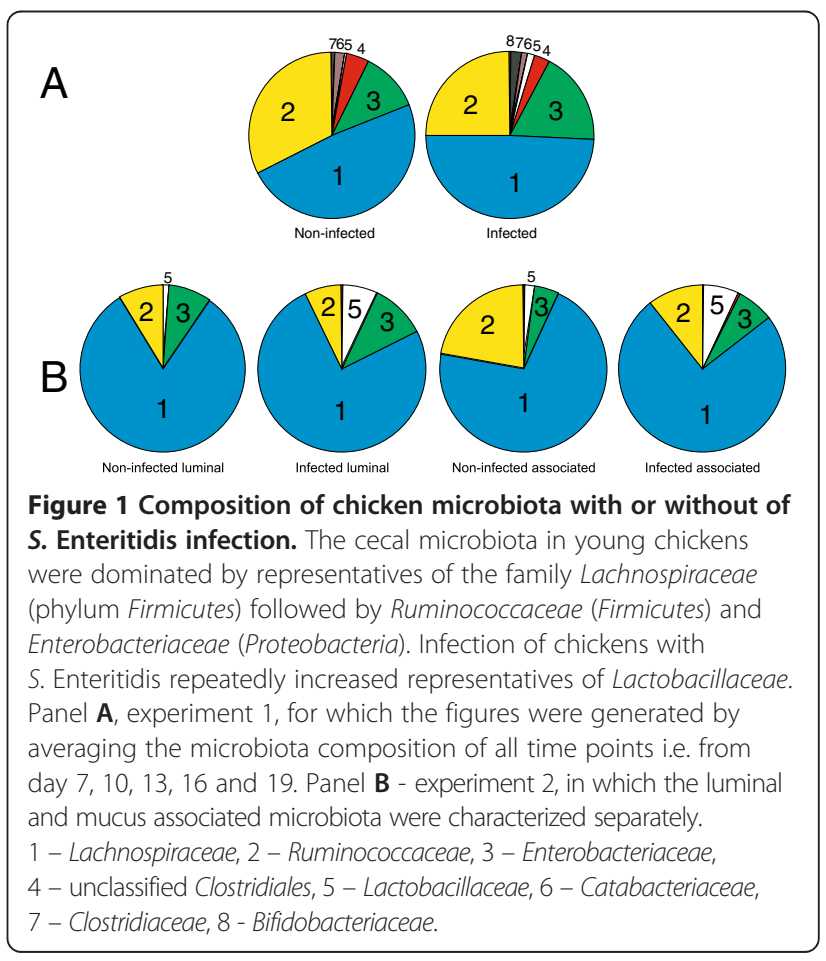



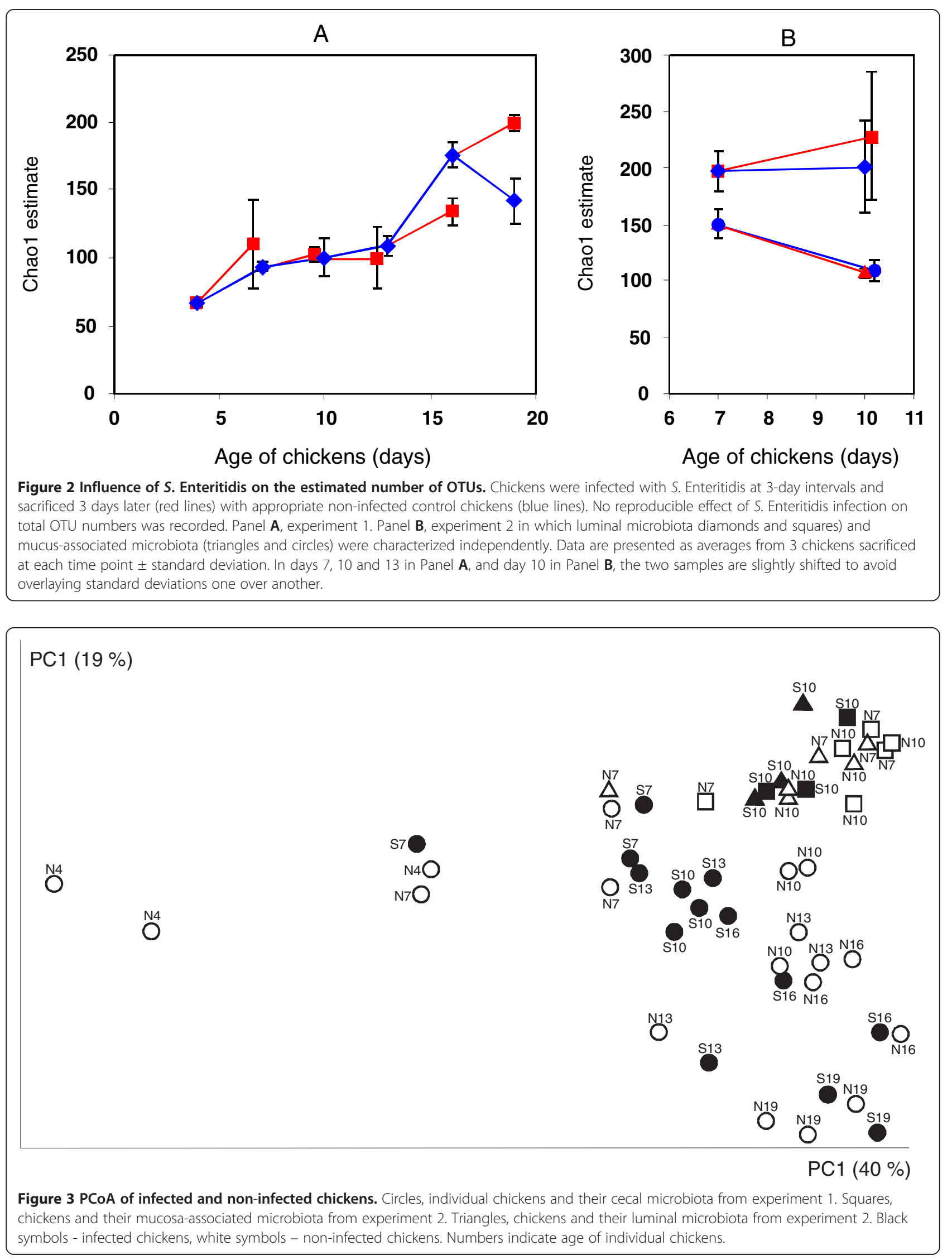


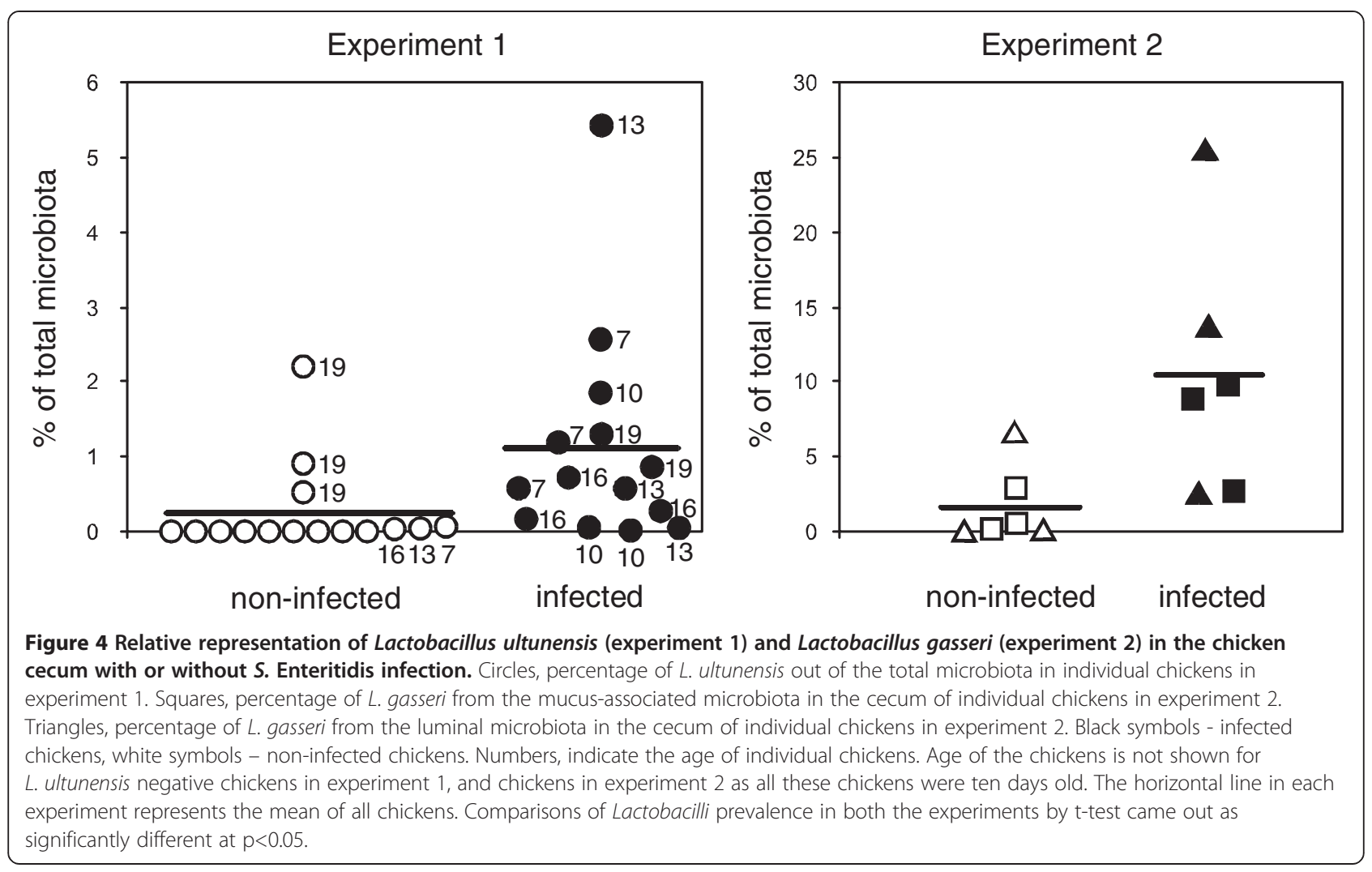

\section{Discussion}

Chicken gut microbiota is understood to be one of the most important components of the host's resistance to Salmonella infection as older chickens are more resistant to Salmonella infection than the younger ones [26,27] and the protective effect of preparations containing gut microbiota from healthy hens has been described [28-31]. In addition, antibiotic therapy in chickens which considerably affects gut microbiota [11] makes the chickens more susceptible to Salmonella infection [32-34]. This clearly points towards the role of the gut microbiota in host resistance and its influence on the maturation of the host immune system. However, it is unclear whether Salmonella infection leads to changes in the microbiota composition resulting in a growth advantage for Salmonella.

In this study we therefore addressed this topic using a model of $S$. Enteritidis and newly hatched chicks. Unlike our expectations but similar to previous reports $[16,35]$, the changes in cecal microbiota after $S$. Enteritidis infection were quite low. The predominant microbiota remained unaffected by the infection as documented by minimal differences in the total number of OTUs before and after the infection. Similarly, no obvious clustering was recorded after PCoA analysis. Even a separate analysis of luminal and mucus-associated microbiota did not show any clear profile although the changes were expected to be more pronounced in mucus-associated microbiota due to the inflammation induced by Salmonella infection and the change in redox status close to the mucosa [21].

Similar to our previous study [16] we noticed that Enterobacteriaceae increased and Ruminococcaceae decreased in both experiments, despite not reaching statistical significance. The only significant difference was the increase of Lactobacillaceae after S. Enteritidis infection. This was rather unexpected, however, we believe that this observation is correct for the following reasons. First, a significant increase in Lactobacillaceae was observed in both experiments. Second, even though Lactobacillaceae was not the predominant family in the chicken cecum, this family still formed around $1 \%$ of the total microbiota and its changes were therefore not observed in the minority population which might be subjected to a greater sample-to-sample variation. Third, the increase in Lactobacillaceae was caused by an increase in the major OTUs of this family in both experiments. This was different from some of the OTUs of Lachnospiraceae or Ruminococcaceae families which showed a significant difference in one of the two experiments but such OTUs always represented a minority of the OTUs within these families. Fourth, the two OTUs of Lactobacillaceae which increased in experiments 1 and 2 belonged to two different species, Lactobacillus ultunensis 
and Lactobacillus gasseri, respectively. The increase of Lactobacillaceae after $S$. Enteritidis infection was therefore not specific for a particular Lactobacillus clone but seems to be general, irrespective of Lactobacillus species. Finally, unlike members of Ruminococcaceae or Lachnospiraceae which are obligate anaerobes, Lactobacilli are microaerophilic bacteria which may allow them to survive under conditions with increased redox potential due to the production of reactive oxygen species by granulocytes infiltrating the site of inflammation $[15,21,36]$.

\section{Conclusions}

Based on our results we conclude that $S$. Enteritidis infection in young chickens influences the microbiota composition, however, the scope of these modifications is minor. Changes in chicken cecal microbiota after $S$. Enteritidis infection can be therefore characterized more as an indirect consequence of the infection rather than a positively selected evolutionary trait.

\section{Methods}

\section{Experimental animals and sample collection}

Male ISA Brown chickens (Hendrix Genetics, Boxmeer, The Netherlands) were obtained from a local commercial hatchery on the day of hatching. The parents of the chickens used in this study were vaccinated against salmonellosis. The chickens were reared in wire cages in the experimental animal house and allowed free access to water and pathogen, antibiotic and coccidiostatic-free feed. Three chickens were sacrificed on day 4, 7, 10, 13, 16 and 19 of life. In addition, 3 chickens were orally infected with $S$. Enteritidis 147 of phage type 4 [36] when aged 4, 7, 10, 13 and 16 days and were sacrificed 3 days post-infection. After the inoculation, the orally infected chickens were housed separately from the noninfected controls. Short segments of cecum together with its contents were collected from both the infected and non-infected chickens and were frozen at $-20^{\circ} \mathrm{C}$ within 10 min after collection.

In the second experiment we sacrificed 7- and 10-day-old chickens, three chickens for each time point. In addition, a group of 3 chickens was orally infected with $S$. Enteritidis when aged 7 days and sacrificed 3 days later. During post mortem sample collection, the cecal contents were squeezed out of the cecum and collected separately. The cecal wall was then washed 3 times in PBS with gentle shaking to remove all luminal bacteria and mucus associated bacteria were collected from the washed cecum by scraping the mucus with a plastic scapula. The cecal contents and mucus-associated microbiota samples were then frozen at $-20^{\circ} \mathrm{C}$ within $10 \mathrm{~min}$ and saved for no longer than 2 months until DNA purification.
All animal treatments and handling have been performed according to current Czech legislation and have been approved by the Ethics Committee of the Czech Ministry of Agriculture.

\section{DNA purification}

After slow defrosting at room temperature, approx. 240-260 mg of total cecal content from experiment 1, and the cecal contents or mucus samples from experiment 2 were homogenized for $1 \mathrm{~min}$ at 7000 RPM in the MagNALyzer (Roche Diagnostics) using zirconia silica beads (BioSpec Products). Following homogenization, the DNA was extracted using a QIAamp DNA Stool Mini Kit (Qiagen) according to the manufacturer's instructions and purified DNA was stored at $-20^{\circ} \mathrm{C}$ until use.

\section{Pyrosequencing}

The purified DNA was used as a template in PCR with the forward primer 5 ' CGTATCGCCTCCCTCGCGCCA TCAG - MID-GGAGGCAGCAGTRRGGAAT 3', and reverse primer 5 ' CTATGCGCCTTGCCAGCCCGCT CAG- MID- CTACCRGGGTATCTAATCC 3' using HotStarTaq Master Mix Kit following instructions of the manufacturer (Qiagen). The underlined sequences were required at different steps of pyrosequencing while those in italics are sequences complementary to the conserved parts of $16 \mathrm{~S}$ rDNA flanking the V3/V4 hypervariable region [37]. The 454 Standard MID Set for sample barcoding was used. Cycling conditions consisted of hot start at $95^{\circ} \mathrm{C}$ for $15 \mathrm{~min}$ followed by 30 cycles of incubation at $94^{\circ} \mathrm{C}$ for $40 \mathrm{~s}, 55^{\circ} \mathrm{C}$ for $55 \mathrm{~s}$ and $72^{\circ} \mathrm{C}$ for $60 \mathrm{~s}$. PCR was terminated by a final extension at $72^{\circ} \mathrm{C}$ for 5 min. After PCR, the amplification products approx. $525 \mathrm{bp}$ in size were separated electrophoretically in a 1.2\% agarose gel, gel-purified using a QIAquick Gel Extraction Kit (Qiagen) and subjected to pyrosequencing. Pyrosequencing was performed using GS Junior Titanium sequencing chemistry and a GS Junior 454 sequencer exactly according to the manufacturer's instructions (Roche). In one sequencing run, the amplification products from 15 to 24 samples were mixed and analyzed.

\section{Sequence analysis}

Fasta and qual files generated as an output of the pyrosequencing were uploaded into Qiime software [38]. Quality trimming criteria included no mismatch in MID sequences and a maximum of 1 mismatch in primer sequences. The obtained sequences with qual score higher than 20 were shortened to the same length of $350 \mathrm{bp}$ and classified with RDP Seqmatch with an OTU discrimination level set to $97 \%$. In the next step, chimeric sequences were predicted and excluded from the analysis. Diversity analyses (rarefaction curves and Chao1 
richness) on OTU clusters were performed both using all sequences available for each sample and using the same number of randomly selected sequences adjusted to the number of sequences available for the sample with the lowest coverage. Finally, UniFrac analysis [39] followed by principal coordinate analysis (PCoA) was used to characterize the diversity in the microbial populations tested. The significance of increase or decrease of particular OTUs was calculated using a t-test comparing the percentage representation of each OTU in microbiomes of all infected and all non-infected chickens, separately for experiment 1 and experiment 2. For this analysis, the microbiomes of 4-day-old, non-infected chickens in experiment 1, and 7-day-old, non-infected chickens in experiment 2 , were excluded from the analysis as these had no age-matched infected counterparts.

\section{Additional file}

Additional file 1: List of all OTUs identified in this study.

\section{Competing interests}

The authors state that they do not have and financial or personal conflicts that could inappropriately bias their work.

\section{Authors' contributions}

PV performed the pyrosequencing, analysed the data and helped to draft the manuscript. MF purified the DNA. FS and $\mathrm{HH}$ were responsible for the animal experiments and sample collection. IR participated in the design of the study, data analysis and helped to draft the manuscript. All authors read and approved the final manuscript.

\section{Acknowledgement}

This work has been supported by EMIDA project HealthyGut, and projects AdmireVet CZ.1.05/2.1.00/01.0006, ED0006/01/01 from the Ministry of Education of the Czech Republic and project 0002716202 from the Ministry of Agriculture of the Czech Republic. Authors wish to thank Peter Eggenhuizen for his English language corrections.

Received: 28 March 2013 Accepted: 3 July 2013

Published: 15 July 2013

\section{References}

1. Gaboriau-Routhiau V, Rakotobe S, Lecuyer E, Mulder I, Lan A, Bridonneau C, Rochet V, Pisi A, De Paepe M, Brandi G, et al: The key role of segmented filamentous bacteria in the coordinated maturation of gut helper $\mathrm{T}$ cell responses. Immunity 2009, 31:677-689.

2. Hill DA, Hoffmann C, Abt MC, Du Y, Kobuley D, Kirn TJ, Bushman FD, Artis D: Metagenomic analyses reveal antibiotic-induced temporal and spatial changes in intestinal microbiota with associated alterations in immune cell homeostasis. Mucosal Immunol 2010, 3:148-158.

3. Ismail AS, Behrendt CL, Hooper LV: Reciprocal interactions between commensal bacteria and gamma delta intraepithelial lymphocytes during mucosal injury. J Immunol 2009, 182:3047-3054.

4. Lillehoj HS: Avian gut-associated immune system: implication in coccidial vaccine development. Poult Sci 1993, 72:1306-1311.

5. Lu J, Idris U, Harmon B, Hofacre C, Maurer JJ, Lee MD: Diversity and succession of the intestinal bacterial community of the maturing broiler chicken. Appl Environ Microbiol 2003, 69:6816-6824.

6. Zhu XY, Zhong T, Pandya Y, Joerger RD: $16 \mathrm{~S}$ rRNA-based analysis of microbiota from the cecum of broiler chickens. Appl Environ Microbiol 2002, 68:124-137.

7. OToole PW, Claesson MJ: Gut microbiota: Changes throughout the lifespan from infancy to elderly. Int Dairy J 2010, 20:281-291.
8. Dethlefsen $L$, Huse $S$, Sogin ML, Relman DA: The pervasive effects of an antibiotic on the human gut microbiota, as revealed by deep 16S rRNA sequencing. PLOS Biol 2008, 6:e280.

9. Lofmark S, Jernberg C, Jansson JK, Edlund C: Clindamycin-induced enrichment and long-term persistence of resistant Bacteroides spp. and resistance genes. J Antimicrob Chemother 2006, 58:1160-1167.

10. Robinson CJ, Young VB: Antibiotic administration alters the community structure of the gastrointestinal micobiota. Gut Microbes 2010, 1:279-284.

11. Videnska P, Faldynova M, Juricova H, Babak V, Sisak F, Havlickova H, Rychlik I: Chicken faecal microbiota and disturbances induced by single or repeated therapy with tetracycline and streptomycin. BMC Vet Res 2013, 9:30.

12. Hoffmann C, Hill DA, Minkah N, Kirn T, Troy A, Artis D, Bushman F: Community-wide response of the gut microbiota to enteropathogenic Citrobacter rodentium infection revealed by deep sequencing. Infect Immun 2009, 77:4668-4678.

13. Lupp C, Robertson ML, Wickham ME, Sekirov I, Champion OL, Gaynor EC, Finlay BB: Host-mediated inflammation disrupts the intestinal microbiota and promotes the overgrowth of Enterobacteriaceae. Cell Host Microbe 2007, 2:119-129.

14. Manichanh C, Rigottier-Gois L, Bonnaud E, Gloux K, Pelletier E, Frangeul L, Nalin R, Jarrin C, Chardon P, Marteau P, et al: Reduced diversity of faecal microbiota in Crohn's disease revealed by a metagenomic approach. Gut 2006, 55:205-211.

15. Barman M, Unold D, Shifley K, Amir E, Hung K, Bos N, Salzman N: Enteric salmonellosis disrupts the microbial ecology of the murine gastrointestinal tract. Infect Immun 2008, 76:907-915.

16. Juricova H, Videnska P, Lukac M, Faldynova M, Babak V, Havlickova H, Sisak $F$, Rychlik I: Influence of Salmonella enterica serovar Enteritidis infection on the development of cecum microbiota in newly hatched chicks. Appl Environ Microbiol 2013, 79:745-7.

17. Stecher B, Robbiani R, Walker AW, Westendorf AM, Barthel M, Kremer M, Chaffron S, Macpherson AJ, Buer J, Parkhill J, et al: Salmonella enterica serovar typhimurium exploits inflammation to compete with the intestinal microbiota. PLOS Biol 2007, 5:2177-2189.

18. Thiennimitr P, Winter SE, Baumler AJ: Salmonella, the host and its microbiota. Curr Opin Microbiol 2012, 15:108-114.

19. Santos RL, Raffatellu M, Bevins CL, Adams LG, Tukel C, Tsolis RM, Baumler AJ: Life in the inflamed intestine, Salmonella style. Trends Microbiol 2009 17:498-506

20. Raffatellu M, George MD, Akiyama Y, Hornsby MJ, Nuccio SP, Paixao TA, Butler BP, Chu H, Santos RL, Berger T, et al: Lipocalin-2 resistance confers an advantage to Salmonella enterica serotype Typhimurium for growth and survival in the inflamed intestine. Cell Host Microbe 2009, 5:476-486.

21. Winter SE, Thiennimitr P, Winter MG, Butler BP, Huseby DL, Crawford RW, Russell JM, Bevins CL, Adams LG, Tsolis RM, et al: Gut inflammation provides a respiratory electron acceptor for Salmonella. Nature 2010, 467:426-429.

22. Berndt A, Wilhelm A, Jugert C, Pieper J, Sachse K, Methner U: Chicken cecum immune response to Salmonella enterica serovars of different levels of invasiveness. Infect Immun 2007, 75:5993-6007.

23. Crhanova M, Hradecka H, Faldynova M, Matulova M, Havlickova H, Sisak F, Rychlik I: Immune response of chicken gut to natural colonization by gut microflora and to Salmonella enterica serovar Enteritidis infection. Infect Immun 2011, 79:2755-2763.

24. Matulova M, Rajova J, Vlasatikova L, Volf J, Stepanova H, Havlickova H, Sisak F, Rychlik I: Characterization of chicken spleen transcriptome after infection with Salmonella enterica serovar Enteritidis. PLoS One 2012 7:e48101.

25. Danzeisen $J L$, Kim HB, Isaacson RE, Tu ZJ, Johnson TJ: Modulations of the chicken cecal microbiome and metagenome in response to anticoccidial and growth promoter treatment. PLoS One 2011, 6:e27949.

26. Beal RK, Powers C, Wigley P, Barrow PA, Smith AL: Temporal dynamics of the cellular, humoral and cytokine responses in chickens during primary and secondary infection with Salmonella enterica serovar Typhimurium. Avian Pathol 2004, 33:25-33.

27. Withanage GS, Wigley P, Kaiser P, Mastroeni P, Brooks H, Powers C, Beal R, Barrow P, Maskell D, McConnell I: Cytokine and chemokine responses associated with clearance of a primary Salmonella enterica serovar Typhimurium infection in the chicken and in protective immunity to rechallenge. Infect Immun 2005, 73:5173-5182. 
28. Endt K, Stecher B, Chaffron S, Slack E, Tchitchek N, Benecke A, Van Maele L, Sirard JC, Mueller AJ, Heikenwalder M, et al: The microbiota mediates pathogen clearance from the gut lumen after non-typhoidal Salmonella diarrhea. PLoS Pathog 2010, 6:e1001097.

29. Hoszowski A, Truszczynski M: Prevention of Salmonella typhimurium caecal colonisation by different preparations for competitive exclusion. Comp Immunol Microbiol Infect Dis 1997, 20:111-117.

30. Methner U, Barrow PA, Martin G, Meyer H: Comparative study of the protective effect against Salmonella colonisation in newly hatched SPF chickens using live, attenuated Salmonella vaccine strains, wild-type Salmonella strains or a competitive exclusion product. Int J Food Microbiol 1997, 35:223-230.

31. Methner U, Barrow PA, Berndt A, Steinbach G: Combination of vaccination and competitive exclusion to prevent Salmonella colonization in chickens: experimental studies. Int J Food Microbiol 1999, 49:35-42

32. Barrow PA: Further observations on the effect of feeding diets containing avoparcin on the excretion of salmonellas by experimentally infected chickens. Epidemiol Infect 1989, 102:239-252.

33. Croswell A, Amir E, Teggatz P, Barman M, Salzman NH: Prolonged impact of antibiotics on intestinal microbial ecology and susceptibility to enteric Salmonella infection. Infect Immun 2009, 77:2741-2753.

34. Sekirov I, Tam NM, Jogova M, Robertson ML, Li Y, Lupp C, Finlay BB: Antibiotic-induced perturbations of the intestinal microbiota alter host susceptibility to enteric infection. Infect Immun 2008, 76:4726-4736.

35. Nordentoft S, Molbak L, Bjerrum L, De Vylder J, Van Immerseel F, Pedersen $K$ : The influence of the cage system and colonisation of Salmonella Enteritidis on the microbial gut flora of laying hens studied by T-RFLP and 454 pyrosequencing. BMC Microbiol 2011, 11:187.

36. Rychlik I, Karasova D, Sebkova A, Volf J, Sisak F, Havlickova H, Kummer V, Imre A, Szmolka A, Nagy B: Virulence potential of five major pathogenicity islands (SPI-1 to SPI-5) of Salmonella enterica serovar Enteritidis for chickens. BMC Microbiol 2009, 9:268.

37. Nossa CW, Oberdorf WE, Yang L, Aas JA, Paster BJ, Desantis TZ, Brodie EL, Malamud D, Poles MA, Pei Z: Design of 165 rRNA gene primers for 454 pyrosequencing of the human foregut microbiome. World I Gastroenterol 2010, 16:4135-4144.

38. Caporaso JG, Kuczynski J, Stombaugh J, Bittinger K, Bushman FD, Costello EK, Fierer N, Pena AG, Goodrich JK, Gordon Jl, et al: QIIME allows analysis of high-throughput community sequencing data. Nat Methods 2010, 7:335-336.

39. Lozupone C, Knight R: UniFrac: a new phylogenetic method for comparing microbial communities. Appl Environ Microbiol 2005, 71:8228-8235.

doi:10.1186/1746-6148-9-140

Cite this article as: Videnska et al:: Influence of Salmonella enterica serovar Enteritidis infection on the composition of chicken cecal microbiota. BMC Veterinary Research 2013 9:140.

\section{Submit your next manuscript to BioMed Central and take full advantage of:}

- Convenient online submission

- Thorough peer review

- No space constraints or color figure charges

- Immediate publication on acceptance

- Inclusion in PubMed, CAS, Scopus and Google Scholar

- Research which is freely available for redistribution 